\title{
The dietary role of unprocessed beef $\&$ lamb in a representative sample of adults aged 18-64 years in Ireland
}

\author{
E. O’Sullivan ${ }^{1}$, L. Kehoe ${ }^{2}$, C. Cocking ${ }^{2}$, B.A. McNulty ${ }^{3}$,A.P. Nugent ${ }^{3,4}$, K. Cashman ${ }^{2}$, \\ J. Walton ${ }^{1,2}$ and A. Flynn ${ }^{2}$ \\ ${ }^{1}$ Department of Biological Sciences, Munster Technological University, Munster, Ireland, \\ ${ }^{2}$ School of Food and Nutritional Sciences, University College Cork, Cork, Ireland, \\ ${ }^{3}$ UCD Institute of Food and Health, University College Dublin, Dublin, Ireland and \\ ${ }^{4}$ Institute for Global Food Security, School of Biological Sciences, Queens University Belfast, UK
}

Meat is a nutrient-dense food that plays a central role in the diet, contributing significantly to intakes of energy, protein and a number of essential micronutrients. However, nutritive quality is variable across meat types and cuts, and dietary guidelines across Europe typically encourage the consumption of lean meats whilst limiting the consumption of processed meats ${ }^{(1)}$. The aim of this analysis was to estimate intakes of unprocessed beef \& lamb and their contribution to energy and nutrient intakes in adults aged 18-64 years in Ireland.

Analyses were based on data from the National Adult Nutrition Survey (NANS) (2008-2010) (n 1500) (www.iuna.net). For this study, data from the sub-group of adults aged 18-64 years (n 1274) were analysed. A 4-day semi-weighed food record was used to collect dietary intake data and energy and nutrient intakes were estimated based on UK and Irish food composition tables ${ }^{(2,3)}$. 'Unprocessed beef \& lamb' was defined as beef/lamb that had not undergone any preserving process other than chilling, freezing or salting, and included beef/lamb dishes that were wrapped in a controlled atmosphere. The mean daily intake (MDI) of 'unprocessed beef and lamb' was estimated following disaggregation of the non-meat components in composite dishes. The contribution of 'unprocessed beef $\&$ lamb' to intakes of energy and nutrients were determined including the non-meat components ${ }^{(4)}$. Statistical analysis was carried out using SPSSC v26.

'Unprocessed beef \& lamb' was consumed by $76 \%$ of adults aged $18-64$ years with beef being more commonly consumed (70\%) than lamb (16\%). Men had a higher MDI of 'unprocessed beef \& lamb' (56 vs 30g), beef (47 vs $26 \mathrm{~g}$ ) and lamb (9 vs $4 \mathrm{~g}$ ) compared to women. 'Unprocessed beef \& lamb' contributed $8 \%$ of the MDI of energy in this population group and contributed greater proportions of protein $(19 \%)$, total fat $(12 \%)$ and MUFA $(13 \%)$ and smaller proportions of PUFA $(5 \%)$, carbohydrate and dietary fibre $(2-3 \%)$. Relative to energy intake, it contributed greater proportions of vitamin D (14\%), niacin (15\%), vitamins B6 (11\%), B12 (30\%), iron $(12 \%)$ and zinc $(27 \%)$, similar proportions of vitamins A and $\mathrm{K}(6-8 \%)$ and smaller proportions of folate and potassium $(4-$ $5 \%$ ). 'Unprocessed beef \& lamb' contributed greater proportions of MDI of saturated fat (13\%), similar proportions of salt ( $9 \%)$ and smaller proportions of total and free sugars (1-2\%).

These findings show that 'unprocessed beef and lamb' is consumed by three-quarters of adults aged 18-64 years in Ireland contributing $8 \%$ of energy intake. 'Unprocessed beef \& lamb' contribute to a number of important nutrients, such as protein, MUFA, vitamin D, niacin, vitamin B6, vitamin B12, iron and zinc. For nutrients for which excess may have potential adverse health effects, 'unprocessed beef and lamb' contribute relatively small proportions of salt, total and saturated fat.

\section{Acknowledgements}

The National Adult Nutrition Survey was funded by the Irish Department of Agriculture, Food and the Marine.

\section{References}

1. Cocking C, et al. (2020) Nutr Res Rev 33(2):181-189.

2. Food Standards Agency (2002) McCance \& Widdowson's The Composition of Foods. Cambridge: London.

3 Black LJ, et al. (2011) J Food Compost Anal 24(7):1017-1023.

4 Krebs-Smith SM, et al. (1989) J Am Diet Assoc 89(5): 671-676. 